\title{
COUNTING 3 BY $n$ LATIN RECTANGLES
}

\author{
K. P. BOGART AND J. Q. LONGYEAR
}

\begin{abstract}
A $k$ by $n$ rectangular array $A$ is called a Latin rectangle if all the integers $1,2, \ldots, n$ appear in each row of $A$ and if $k$ distinct integers occur in each column of $A$. The number of $k$ by $n$ Latin rectangles is unknown for $k \geqslant 4$; Riordan has given a formula for the number of 3 by $n$ rectangles in terms of the solutions of the derangement (or displacement) problem and the menage problem. In this paper we derive an elementary formula for the number of 3 by $n$ Latin rectangles by using Möbius inversion.

We include a table giving the approximate number of 3 by $n$ Latin rectangles for $n \leqslant 20$. The table has exact values for $n \leqslant 11$.
\end{abstract}

1. Introduction. A $k$ by $n$ Latin rectangle or Youden design is a $k$ by $n$ matrix with the properties that each row contains all the integers $1,2, \ldots, n$ and each column contains $k$ distinct integers. If $L(k, n)$ is the number of $k$ by $n$ Latin rectangles, then $L(k, n)$ is unknown when $4 \leqslant k \leqslant n$ except for some specific values of $n$. It is known that $L(1, n)=n !$ and that $L(2, n)=[1 / 2+$ $n ! / e]$ where the square brackets denote the greatest integer function. Riordan [4] gives several elegant formulae for $L(3, n)$ in terms of menage numbers, displacement numbers and various recursions.

In this paper we obtain an elementary formula for $L(3, n)$ by using Möbius inversion on a suitable lattice. We append a table giving the exact value of $L(3, n)$ for $n \leqslant 11$ and the approximate values for $n \leqslant 20$. This table was prepared by C. W. Kaufman and F. L. Toher, Jr., using the Dartmouth Time Sharing System and the language COMPASS written by Mr. Kaufman.

For a discussion of Möbius inversion, see Rota [5].

2. Preliminary discussion. We denote sets by Roman capitals and their cardinalities by the corresponding lower case letter, thus $X$ is a set of cardinality $x$. Rather than beginning with Latin rectangles, we consider the collection $e$ of all 3 by $n$ arrays of integers in which each row is a permutation of $1,2, \ldots, n$. We associate with each 3 by $n$ array in $C$ an ordered triple of sets of columns. The set $E_{i j}$ for $1 \leqslant i<j \leqslant 3$ is the set of all columns for which row $i$ and row $j$ have the same entry. The array then uniquely determines the triple of sets $\left(E_{12}, E_{13}, E_{23}\right)$. Those arrays whose triple is $(\phi, \phi, \phi)$ are Latin rectangles. Not every triple of sets of columns $(A$, $B, C)$ can be obtained in this manner since the following condition obviously holds for the "equality triples":

$$
E_{i j} \cap E_{j k}=E_{12} \cap E_{13} \cap E_{23} .
$$

Received by the editors May 31, 1973 and, in revised form, March 6, 1975.

AMS (MOS) subject classifications (1970). Primary 05A15, 15B15; Secondary 06A15. 
It can be shown that the equality triples form a lattice $L$ under the relation $\leqslant$ defined by $\left(B_{1}, B_{2}, B_{3}\right) \leqslant\left(A_{1}, A_{2}, A_{3}\right)$ iff each $B_{i}$ is a subset of $A_{i}$. If we let $f\left(A_{1}, A_{2}, A_{3}\right)$ be the number of arrays having $\left(A_{1}, A_{2}, A_{3}\right)$ as equality triple, and let $g\left(B_{1}, B_{2}, B_{3}\right)$ be the number of arrays whose equality triple contains $\left(B_{1}, B_{2}, B_{3}\right)$, then

$$
g\left(B_{1}, B_{2}, B_{3}\right)=\sum f\left(A_{1}, A_{2}, A_{3}\right)
$$

where the sum runs over all triples $\left(A_{1}, A_{2}, A_{3}\right) \geqslant\left(B_{1}, B_{2}, B_{3}\right)$. Thus, as in [5], we may use the Möbius function $\mu$ of the lattice $L$ to obtain the following formula:

$$
f\left(A_{1}, A_{2}, A_{3}\right)=\sum \mu\left((\phi, \phi, \phi),\left(B_{1}, B_{2}, B_{3}\right)\right) g\left(B_{1}, B_{2}, B_{3}\right) .
$$

The sum runs over all triples $\left(B_{1}, B_{2}, B_{3}\right) \geqslant\left(A_{1}, A_{2}, A_{3}\right)$. In particular, if $\left(A_{1}, A_{2}, A_{3}\right)=(\phi, \phi, \phi)$, we have

$$
L(3, n)=f(\phi, \phi, \phi)=\sum \mu\left((\phi, \phi, \phi),\left(B_{1}, B_{2}, B_{3}\right)\right) g\left(B_{1}, B_{2}, B_{3}\right) .
$$

The sum is over all equality triples. Thus we need to determine $g$ and compute $\mu$.

3. Deriving the Möbius function of $L$. Crapo [2] has shown that if $M$ is a locally finite lattice with a closure relation, and if $N$ is the ordered set of closed elements of $M$, then, denoting the corresponding Möbius functions by $\mu_{M}$ and $\mu_{N}$

$$
\mu_{N}(x, y)=\sum \mu_{M}(x, z) .
$$

(Bogart [1] and Greene [3] have shown that this result holds if the word poset is substituted for lattice. Greene's proof is particularly nice.) Here the sum runs over all elements $z$ in $M$ such that the closure of $z$ is $y$. In order to apply (4) with $N=L$, we need a lattice $M$ with a known or easily derived Möbius function, and a closure $\gamma$ from $M$ to $L$. We use the lattice of all triples of sets of columns for $M$, since this has the well-known Möbius function $\mu_{3}$ given by

$$
\mu_{3}\left(\left(A_{1}, A_{2}, A_{3}\right),\left(B_{1}, B_{2}, B_{3}\right)\right)=(-1)^{t}
$$

where $t=a_{1}-b_{1}+a_{2}-b_{2}+a_{3}-b_{3}$.

For our closure relation we let

$$
\gamma(A, B, C)=(A \cup(B \cap C), B \cup(A \cap C), C \cup(A \cap B)) .
$$

It is not difficult to verify that $\gamma$ is a closure in the sense that $\gamma$ satisfies the usual properties for a closure, namely

(i) $\gamma(A, B, C) \leqslant(A, B, C)$,

(ii) $\gamma^{2}(A, B, C)=\gamma(A, B, C)$,

(iii) if $(A, B, C) \leqslant(D, E, F)$ then $\gamma(A, B, C) \leqslant \gamma(D, E, F)$.It is also easy to verify that a triple is $\gamma$-closed if and only if it is an equality triple. We must determine exactly which sets close to a given equality triple in order to compute the Möbius function $\mu$ of $L$ from $\mu_{3}$.

Let $(U, V, W)$ be an equality triple with $U \cap V \cap W=S$, and suppose that $\gamma(X, Y, Z)=(U, V, W)$. Then 


$$
\begin{aligned}
U & =X \cup(Y \cap Z) \subseteq X \cup(V \cap W)=X \cup(U \cap V \cap W) \\
& =X \cup S \subseteq U \cup S=U .
\end{aligned}
$$

Thus $U=X \cup S$. Similarly, $V=Y \cup S$ and $W=Z \cup S$. These three equations are not sufficient to insure that the closure of $(X, Y, Z)$ is the triple $(U, V, W)$, for if $c$ were in $S$ but not in $Y \cup Z$, then $\gamma(X, Y, Z)$ would not be $(U, V, W)$. Thus every $c$ in $S$ must be contained in at least two of the sets $X$, $Y$ and $Z$.

If the triple $(X, Y, Z)$ has the property that each element of $S$ is in at least two sets in the triple and satisfies the equations $U=X \cup S, V=Y \cup S$, and $W=Z \cup S$, then $(X, Y, Z)$ closes to $(U, V, W)$.

Now we determine how many triples have $(U, V, W)$ as their closure. Select any subset $T$ of $S$ to be the set of those elements of $S$ to be contained in exactly two members of a triple closing to $(U, V, W)$. Each of the $3^{t}$ functions $\lambda$ from $T$ to $\{1,2,3\}$ gives rise to a unique decomposition $B_{1}=\lambda^{-1}(1), B_{2}=\lambda^{-1}(2), B_{3}=\lambda^{-1}(3)$ of $T$, and conversely, so that there are exactly $3^{t}$ possible triples closing to $(U, V, W)$ defined by the equations in (5).

$$
X=U-B_{1}, \quad Y=V-B_{2}, \quad Z=W-B_{3} .
$$

Thus for each $T \subseteq S$, there are $3^{t}$ triples closing to $(U, V, W)$, and we may rewrite (4) in the case that $x$ is the triple of empty sets (which is closed) and the $z$ 's are the triples $(X, Y, Z)$ closing to $(U, V, W)$ as

$$
\mu((\phi, \phi, \phi),(U, V, W))=\sum 3^{t}(-1)^{u+v+w-t}
$$

where the sum ranges over all subsets $T$ of $S=U \cap V \cap W$. This gives

$$
\begin{aligned}
\mu((\phi, \phi, \phi),(U, V, W)) & =\sum_{t=0}^{s}\left(\begin{array}{l}
s \\
t
\end{array}\right) 3^{t}\left(-1^{t}\right)(-1)^{u+v+w} \\
& =(-1)^{u+v+w}(1-3)^{s}=(-1)^{u+v+w}(-2)^{s} \\
& =(-1)^{u+v+w+s}(2)^{s} .
\end{aligned}
$$

Substituting (7) into (3) we obtain the following expression for the number of $3 \times n$ Latin rectangles:

$$
L(3, n)=f(\phi, \phi, \phi)=\sum(-1)^{u+v+w+s}(2)^{s} g(U, V, W)
$$

where the sum is over all equality triples $(U, V, W)$, and $s=|U \cap V \cap W|$.

4. Computing $g(U, V, W)$ and the number of latin rectangles. To compute $g(U, V, W)$, let

$$
S=U \cap V \cap W, \quad P=U-S, \quad Q=V-S, \quad R=W-S .
$$

Thus $(-1)^{u+v+w+s}=(-1)^{u+v+w-3 s}=(-1)^{p+q+r}$. The number of ordered triples $(U, V, W)$ with given values of $p, q, r$ and $s$ is the multinomial coefficient $C(n ; p, q, r, s, d)$ where $d=n-(p+q+r+s)$ since $P, Q, R$ and $S$ are pairwise disjoint. Let $N$ denote the set of the $n$ columns of our array. Let $D$ be the columns not in $P, Q, R$ or $S$, i.e., $D=N-(P \cup Q \cup$ $R \cup S)$. We can rewrite (8) as

$$
L(3, n)=f(\phi, \phi, \phi)=\sum(-1)^{p+q+r}(2)^{s} g(U, V, W) C(n ; p, q, r, s, d)
$$


where the sum is over all possible sets of parameters $(p, q, r, s, d)$ and $(U, V$, $W$ ) is any equality triple with these parameters. (Note that the parameters uniquely determine $g(U, V, W)$.)

We now compute $g(U, V, W)$ for a given parameter set and fixed $U, V$ and $W$ with these parameters. First select any set of $s$ integers between 1 and $n$ to go in the columns of $S=U \cap V \cap W$. There are $s$ ! ways of arranging these integers among the columns. So far we have filled in all three rows of our array in the columns of $S$. Now the columns of $P$ are where rows 1 and 2 agree; we select $p$ integers from the $n-s$ integers not in the columns of $S$ and arrange them in the columns of $P$ in rows 1 and 2 in $p$ ! ways. The columns of $Q$ are where rows 1 and 3 agree. We select $q$ integers from the $n-s-p$ remaining integers and arrange them in the columns of $Q$ in row 1 and row 3 in $q$ ! ways. The columns of $R$ are where rows 2 and 3 agree. We select $r$ integers from the $n-s-p-q$ remaining ones and arrange them in the columns of $R$ in rows 2 and 3 in $r$ ! ways. There are

$$
(q+d) !=(n-p-r-s) !
$$

ways to arrange the remaining elements of row $1,(r+d)$ ! ways to arrange the remaining elements of row 2 and $(p+d)$ ! ways to arrange the remaining elements of row 3 . This gives

$$
\begin{aligned}
g(U, V, W)= & \left(\begin{array}{l}
n \\
s
\end{array}\right) s !\left(\begin{array}{c}
n-s \\
p
\end{array}\right) p !\left(\begin{array}{c}
n-s-p \\
q
\end{array}\right) q !\left(\begin{array}{c}
n-p-q-s \\
r
\end{array}\right) r ! \\
& \times(q+d) !(r+d) !(p+d)^{\prime} \\
= & n !(q+d) !(r+d) !(p+d) ! / d ! .
\end{aligned}
$$

By substituting (10) into (9) we obtain

$$
\begin{aligned}
L(3, n)= & \sum(-1)^{p+q+r}(2)^{s} n !(q+d) !(r+d) !(p+d) ! \\
& \times C(n ; p, q, r, s, d) / d ! \\
= & \sum(-1)^{p+q+r}(2)^{s}(n !)^{2} \\
& \times(q+d) !(r+d) !(p+d) ! /(d !)^{2} p ! q ! r ! s !
\end{aligned}
$$

where the sum is over all ordered partitions or compositions $(p, q, r, s, d)$ of $n$.

Values of $N(3, n)$ for $3 \leqslant n \leqslant 20$. To allow accurate computation for large values of $n$, we computed $N(3, n) /(n !)$, the number of Latin rectangles with first row $1,2,3, \ldots, n$.

$\begin{array}{rl}\frac{n}{3} & \frac{N(3, n) / n !}{2} \\ 4 & 24 \\ 5 & 552 \\ 6 & 21,280 \\ 7 & 103,760 \\ 8 & 70,229,264 \\ 9 & 5,792,853,248 \\ 10 & 587,159,944,704 \\ 11 & 71,822,743,499,520\end{array}$




$\begin{array}{cc}\text { Approximate VALUES For } & N(3, n) / n ! \text { FOR } 12 \leqslant n \leqslant 20 \\ 12 & 1.04352735037 \times 10^{16} \\ 13 & 1.77678070051 \times 10^{18} \\ 14 & 3.50461958857 \times 10^{20} \\ 15 & 7.92840412826 \times 10^{22} \\ 16 & 2.03927654048 \times 10^{25} \\ 17 & 5.91793423080 \times 10^{27} \\ 18 & 1.92442722632 \times 10^{30} \\ 19 & 6.96979289286 \times 10^{32} \\ 20 & 2.79603955401 \times 10^{35}\end{array}$

\section{REFERENCES}

1. K. P. Bogart, Closure relations and Möbius functions (submitted).

2. H. Crapo, Möbius inversion in lattices, Arch. Math. (Basel) 19 (1968), 595-607 (1969). MR 39 \#6791.

3. C. Greene, Combinatorial geometry, Notes from the course given at N.S.F. Seminar in Combinatorics at Bowdoin College in 1971.

4. J. Riordan, An introduction to combinatorial analysis, Wiley Publications in Math. Statistics, Wiley, New York; Chapman \& Hall, London, 1958. MR 20 \#3077.

5. G.-C. Rota, On the foundations of combinatorial theory. I, Theory of Möbius functions, Z. Wahrscheinlichkeitstheorie und Verw. Gebiete 2 (1964), 340-368. MR 30 \#4688.

DEPARTMENT OF MATHEMATICS, DARTMOUTH COLLEGE, HANOVER, NEW HAM PSHIRE 03755

DEPARTMENT OF MATHEMATICS, WAYNE STATE UNIVERSITY, DETROIT, MICHIGAN 48202 